Volume 133, Number 7 , Pages 1965-1976

S 0002-9939(05)07885-8

Article electronically published on February 24, 2005

\title{
RODRIGUES TYPE FORMULA FOR ORTHOGONAL POLYNOMIALS ON THE UNIT BALL
}

\author{
YUAN XU
}

(Communicated by Jonathan M. Borwein)

\begin{abstract}
For a class of weight functions invariant under reflection groups on the unit ball, a family of orthogonal polynomials is defined via a Rodrigues type formula using the Dunkl operators. Their properties and their relation with several other bases are explored.
\end{abstract}

\section{INTRODUCTION}

Let $\Pi^{d}=\mathbb{R}\left[x_{1}, \ldots, x_{d}\right]$ denote the space of polynomials in $d$ variables and $\Pi_{n}^{d}$ denote the subspace of polynomials of degree at most $n$. We will use the standard multi-index notation. For $\alpha \in \mathbb{N}_{0}^{d}$ and $x \in \mathbb{R}^{d}, x^{\alpha}=x_{1}^{\alpha_{1}} \cdots x_{d}^{\alpha_{d}}$ is a monomial of degree $|\alpha|=\alpha_{1}+\ldots+\alpha_{d}$.

The classical orthogonal polynomials on the unit ball $B^{d}=\{x:\|x\| \leq 1\}$, where $\|x\|$ is the Euclidean norm of $x$, are orthogonal with respect to the weight function

$$
W_{\mu}(x)=\left(1-\|x\|^{2}\right)^{\mu-1 / 2}, \quad x \in B^{d}, \quad \mu>-1 / 2 .
$$

The study of these polynomials can be traced back to Hermite; see [1] and [5] Chapt. 12]. Among the first orthogonal bases on $B^{d}$ being studied is the one given by the Rodrigues type formula

$$
U_{\alpha}(x)=\left(1-\|x\|^{2}\right)^{-\mu+1 / 2} \partial^{\alpha}\left(1-\|x\|^{2}\right)^{n+\mu-1 / 2}, \quad \alpha \in \mathbb{N}_{0}^{d},
$$

where $\partial_{i}=\partial / \partial x_{i}$ and $\partial^{\alpha}=\partial_{1}^{\alpha_{1}} \cdots \partial_{d}^{\alpha_{d}}$, and a family of polynomials that are biorthogonal to $U_{\alpha}$.

The purpose of this paper is to consider the analogues of $U_{\alpha}$ for orthogonal polynomials on $B^{d}$ with respect to a class of weight functions invariant under a reflection group. We first define the weight function. Let $G$ be a finite reflection group with a fixed positive root system $R_{+}$. Let $\sigma_{v}$ denote the reflection along $v \in R_{+}$, that is, $x \sigma_{v}=x-2\langle x, v\rangle /\|v\|^{2}$ for $x \in \mathbb{R}^{d}$ with the inner product $\langle x, y\rangle=\sum_{i=1}^{d} x_{i} y_{i}$. Thus, $G$ is a finite orthogonal group generated by the reflections $\left\{\sigma_{v}: v \in R_{+}\right\}$. Let $\kappa$ be a multiplicity function defined on $R_{+}$, that is, $\kappa: R_{+} \mapsto \mathbb{R}$

Received by the editors February 13, 2003.

2000 Mathematics Subject Classification. Primary 33C50, 42C10.

Key words and phrases. Orthogonal polynomials on a unit ball, Rodrigues type formula, biorthogonal polynomials, reflection invariant weight function.

This work was partially supported by the National Science Foundation under Grant DMS0201669. 
is a $G$-invariant function. We assume that $\kappa(v) \geq 0$ for all $v \in R_{+}$. Then the function

$$
h_{\kappa}(x)=\prod_{v \in R_{+}}|\langle x, v\rangle|^{\kappa(v)}, \quad x \in \mathbb{R}^{d},
$$

is a positive homogeneous $G$-invariant function of order $\gamma:=\gamma_{\kappa}=\sum_{v \in R_{+}} \kappa_{v}$. For example, if $G$ is the symmetric group, then $h_{\kappa}(x)=\prod_{1 \leq i<j \leq d}\left|x_{i}-x_{j}\right|^{\kappa}$ and $\gamma=d(d-1) \kappa / 2$. In this paper we consider the reflection invariant weight function $W_{\kappa, \mu}$ on the unit ball

$$
W_{\kappa, \mu}(x)=h_{\kappa}^{2}(x)\left(1-\|x\|^{2}\right)^{\mu-1 / 2}, \quad x \in B^{d}, \quad \mu>-1 / 2
$$

where $h_{\kappa}^{2}$ is as in (1.3). If $\kappa=0$, then $W_{\kappa, \mu}(x)$ becomes $W_{\mu}(x)$ in (1.1).

The weight function $h_{\kappa}$ was introduced by Dunkl for the purpose of studying the orthogonal structure for polynomials on the unit sphere $S^{d-1}=\left\{x \in \mathbb{R}^{d}:\|x\|=1\right\}$ with respect to the measure $h_{\kappa}^{2}(x) d \omega$, where $d \omega$ is the rotation invariant measure on $S^{d-1}$. The main ingredient in the study is a family of commuting differentialdifference operators, $\mathcal{D}_{i}$, called Dunkl's operators ([2]),

$$
\mathcal{D}_{i} f(x)=\partial_{i} f(x)+\sum_{v \in R_{+}} \kappa_{v} \frac{f(x)-f\left(x \sigma_{v}\right)}{\langle x, v\rangle}\left\langle e_{i}, v\right\rangle, \quad 1 \leq i \leq d,
$$

where $e_{i}=(0, \ldots, 0,1,0, \ldots, 0)$ with 1 in the $i$-th component. The set $\left\{\mathcal{D}_{i}: 1 \leq\right.$ $i \leq d\}$ generates a commutative algebra of operators containing the $h$-Laplacian $\Delta_{h}=\mathcal{D}_{1}^{2}+\ldots+\mathcal{D}_{d}^{2}$. Let $\mathcal{P}_{n}^{d}$ denote the space of homogeneous polynomials of degree $n$ in $d$ variables. If $p \in \mathcal{P}_{n}^{d}$, then

$$
\int_{S^{d-1}} p(x) q(x) h^{2}(x) d \omega=0, \quad \forall q \in \Pi_{n-1}^{d},
$$

if and only if $\Delta_{h} p=0$. The space $\mathcal{H}_{n}^{d}\left(h_{\kappa}^{2}\right):=\mathcal{P}_{n}^{d} \cap$ ker $\Delta_{h}$ is called the space of $h$ harmonic polynomials of degree $n$. If $\kappa(v)=0$, it agrees with the space of ordinary harmonic polynomials $\mathcal{H}_{n}^{d}:=\mathcal{P}_{n}^{d} \cap \operatorname{ker} \Delta$, where $\Delta$ denote the ordinary Laplacian $\Delta=\partial_{1}^{2}+\ldots+\partial_{d}^{2}$. For the extensive study of $h$-harmonics and the related work, see [4] and the references therein.

The theory of $h$-harmonics has also made it possible to study the orthogonal structure of polynomials with respect to $W_{\kappa, \mu}$ on $B^{d}$. Initial work in this direction has been carried out in [12, 13], which has led to new results even in the case of the classical weight function $W_{\mu}$. In the present paper we show that the orthogonal polynomials $U_{\alpha}(x)$ in (1.2) defined by the Rodrigues type formula can be extended to the reflection invariant weight function $W_{\kappa, \mu}$. That is, we define $U_{\alpha}$ as follows:

Definition 1.1. For $\alpha \in \mathbb{N}_{0}^{d}$, define

$$
U_{\alpha}(x)=\left(1-\|x\|^{2}\right)^{-\mu+1 / 2} \mathcal{D}^{\alpha}\left(1-\|x\|^{2}\right)^{|\alpha|+\mu-1 / 2} .
$$

We will show that $U_{\alpha}(x)$ so defined are orthogonal polynomials with respect to $W_{\kappa, \mu}$ on $B^{d}$. Properties of these polynomials and their relations with other orthogonal bases will be discussed. Let us mention that recently Dunkl and Dunkl-Cherednik operators have been used to give the Rodrigues type formula for nonsymmetric Hermite and Laguerre polynomials with respect to reflection invariant weight functions $([8,10])$ and for Macdonald's polynomials ([7]). 


\section{RODRIGUES TYPE FORMUlA AND ORTHOGONAL BASES}

For $n \in \mathbb{N}_{0}^{d}$ let $\mathcal{V}_{n}^{d}\left(W_{\kappa, \mu}\right)$ denote the subspace of polynomials of degree exactly $n$ in $\Pi^{d}$ that are orthogonal with respect to $W_{\kappa, \mu}$. A polynomial $p \in \mathcal{V}_{n}^{d}\left(W_{\kappa, \mu}\right)$ if $p \in \Pi_{n}^{d}$ and $\int_{B^{d}} p(x) q(x) W_{\kappa, \mu}(x) d x=0$ for all $q \in \Pi_{n-1}^{d}$. We show $U_{\alpha} \in \mathcal{V}_{n}^{d}\left(W_{\kappa, \mu}\right)$.

The definition of $U_{\alpha}$ in Definition 1.1 shows that it depends on $\kappa$ and $\mu$. In the following proposition, we write $U_{\alpha}^{\mu}:=U_{\alpha}$ to emphasize its dependence on $\mu$.

Proposition 2.1. Let $\alpha \in \mathbb{N}_{0}^{d}$ and $|\alpha|=n$. Then $U_{\alpha}^{\mu}$ is a polynomial of degree $n$, and we have a recursive formula

$$
U_{\alpha+e_{i}}^{\mu}(x)=-(2 \mu+1) x_{i} U_{\alpha}^{\mu+1}(x)+\left(1-\|x\|^{2}\right) \mathcal{D}_{i} U_{\alpha}^{\mu+1}(x) .
$$

Proof. Since $\left(1-\|x\|^{2}\right)^{a}$ is invariant under the reflection group, a simple computation shows that

$$
\begin{aligned}
\mathcal{D}_{i} U_{\alpha}^{\mu+1}(x)= & \partial_{i}\left[\left(1-\|x\|^{2}\right)^{-\mu-1 / 2}\right] \mathcal{D}^{\alpha}\left(1-\|x\|^{2}\right)^{|\alpha|+\mu+1 / 2} \\
& +\left(1-\|x\|^{2}\right)^{-\mu-1 / 2} \mathcal{D}^{\alpha+e_{i}}\left(1-\|x\|^{2}\right)^{|\alpha|+\mu+1 / 2} \\
= & (2 \mu+1) x_{i}\left(1-\|x\|^{2}\right)^{-1} U_{\alpha}^{\mu+1}(x)+\left(1-\|x\|^{2}\right)^{-1} U_{\alpha+e_{i}}^{\mu}(x),
\end{aligned}
$$

which proves the recursive relation. That $U_{\alpha}^{\mu}$ is a polynomial of degree $|\alpha|$ is a consequence of this relation (use induction if necessary).

The following proposition plays a key role in proving that $U_{\alpha}$ is in fact an orthogonal polynomial with respect to $W_{\kappa, \mu}$ on $B^{d}$.

Proposition 2.2. Assume that $p$ and $q$ are continuous functions and $p$ vanishes on the boundary of $B^{d}$. Then

$$
\int_{B^{d}} \mathcal{D}_{i} p(x) q(x) h_{\kappa}^{2}(x) d x=-\int_{B^{d}} p(x) \mathcal{D}_{i} q(x) h_{\kappa}^{2}(x) d x .
$$

Proof. The proof is similar to the proof of Lemma 3.7 of [3] (Theorem 5.1.18 of [4]). We shall be brief. Assume $\kappa_{v} \geq 1$. Analytic continuation can be used to extend the range of validity to $\kappa_{v} \geq 0$. Integration by parts shows

$$
\begin{aligned}
& \int_{B^{d}} \partial_{i} p(x) q(x) h_{\kappa}^{2}(x) d x=-\int_{B^{d}} p(x) \partial_{i}\left(q(x) h_{\kappa}^{2}(x)\right) d x \\
& =-\int_{B^{d}} p(x) \partial_{i} q(x) h_{\kappa}^{2}(x) d x-2 \int_{B^{d}} p(x) q(x) h_{\kappa}(x) \partial_{i} h_{\kappa}(x) d x .
\end{aligned}
$$

Let $D_{i}$ denote the difference part of $\mathcal{D}_{i}, \mathcal{D}_{i}=\partial_{i}+D_{i}$. For a fixed root $v$, a simple computation shows that

$$
\int_{B^{d}} D_{i} p(x) q(x) h_{\kappa}^{2}(x) d x=\int_{B^{d}} p(x) \sum_{v \in R_{+}} \kappa_{i} v_{i} \frac{q(x)+q\left(x \sigma_{v}\right)}{\langle x, v\rangle} h_{\kappa}^{2}(x) d x .
$$

Adding these two equations and using the fact that

$$
h_{\kappa}(x) \partial_{i} h_{\kappa}(x)=\sum_{v \in R_{+}} \kappa_{v} v_{i} \frac{1}{\langle v, x\rangle} h_{\kappa}^{2}(x)
$$

completes the proof.

Theorem 2.3. For $\alpha \in \mathbb{N}_{0}^{d}$, the polynomials $U_{\alpha}$ are elements in $\mathcal{V}_{|\alpha|}^{d}\left(W_{\kappa, \mu}\right)$. 
Proof. Let $n=|\alpha|$. For each $\beta \in \mathbb{N}_{0}^{d}$ and $|\beta|<n$, we claim that

$$
\mathcal{D}^{\beta}\left(1-\|x\|^{2}\right)^{n+\mu-1 / 2}=\left(1-\|x\|^{2}\right)^{n-|\beta|+\mu-1 / 2} Q_{\beta}(x)
$$

for some $Q_{\beta} \in \Pi_{n}^{d}$. This follows from induction. The case $\beta=0$ is trivial with $Q_{0}(x)=1$. Suppose the equation is true for $|\beta|<n-1$. Then

$$
\begin{aligned}
& \mathcal{D}^{\beta+e_{i}}\left(1-\|x\|^{2}\right)^{n+\mu-1 / 2}=\mathcal{D}_{i}\left[\left(1-\|x\|^{2}\right)^{n-|\beta|+\mu-1 / 2} Q_{\beta}(x)\right] \\
& =\partial_{i}\left[\left(1-\|x\|^{2}\right)^{n-|\beta|+\mu-1 / 2}\right] Q_{\beta}(x)+\left(1-\|x\|^{2}\right)^{n-|\beta|+\mu-1 / 2} \mathcal{D}_{i} Q_{\beta}(x) \\
& =\left(1-\|x\|^{2}\right)^{n-|\beta|+\mu-3 / 2}\left[-2(n-|\beta|+\mu-1 / 2) x_{i} Q_{\beta}(x)+\left(1-\|x\|^{2}\right) \mathcal{D}_{i} Q_{\beta}(x)\right] \\
& =\left(1-\|x\|^{2}\right)^{n-\left|\beta+e_{i}\right|+\mu-1 / 2} Q_{\beta+e_{i}}(x),
\end{aligned}
$$

where $Q_{\beta+e_{i}}$, as defined above, is clearly a polynomial of degree $|\beta|+1$, which completes the inductive proof. This formula shows, in particular, that the function $\mathcal{D}^{\beta}\left(1-\|x\|^{2}\right)^{n+\mu-1 / 2}$ vanishes on the boundary of $B^{d}$ if $|\beta|<n$. For any polynomial $p \in \Pi_{n-1}^{d}$, using Proposition 2.2 repeatedly gives

$$
\begin{gathered}
\int_{B^{d}} U_{\alpha}(x) p(x) h_{\kappa}^{2}(x)\left(1-\|x\|^{2}\right)^{\mu-1 / 2} d x=\int_{B^{d}} \mathcal{D}^{\alpha}\left[\left(1-\|x\|^{2}\right)^{n+\mu-1 / 2}\right] p(x) h_{\kappa}^{2}(x) d x \\
=(-1)^{n} \int_{B^{d}} \mathcal{D}^{\alpha} p(x)\left(1-\|x\|^{2}\right)^{n+\mu-1 / 2} h_{\kappa}^{2}(x) d x=0,
\end{gathered}
$$

since $\mathcal{D}_{i}: \mathcal{P}_{n}^{d} \mapsto \mathcal{P}_{n-1}^{d}$, which implies $\mathcal{D}^{\alpha} p(x)=0$ as $p \in \Pi_{n-1}^{d}$.

By definition, $U_{\alpha}$ is orthogonal to polynomials of lower degree. However, if $|\alpha|=|\beta|, U_{\alpha}$ and $U_{\beta}$ are not necessarily orthogonal. In fact, they are not and there is another family of orthogonal polynomials in $\mathcal{V}_{n}^{d}\left(W_{\kappa, \mu}\right)$ that are biorthogonal to $U_{\alpha}$. For its definition, we need the projection operator $\operatorname{proj}_{n}^{B}: \mathcal{P}_{n}^{d} \mapsto \mathcal{V}_{n}^{d}\left(W_{\kappa, \mu}\right)$. This operator has an explicit formula ([12]). For $p \in \mathcal{P}_{n}^{d}$,

$$
\operatorname{proj}_{B^{d}} p(x)=\sum_{0 \leq j \leq n / 2} \frac{1}{4^{j} j !(-n-\lambda-\mu+1)_{j}} \Delta_{h}^{j} p(x),
$$

where $\lambda=\gamma_{\kappa}+(d-1) / 2$ and $(a)_{j}=a(a+1) \ldots(a+j-1)$ is the Pochhammer symbol. Let $V_{\kappa}$ be the intertwining operator between the family of partial derivatives and Dunkl's operators, which is a linear operator uniquely determined by ([3])

$$
V_{\kappa} 1=1, \quad V_{\kappa} \mathcal{P}_{n}^{d}=\mathcal{P}_{n}^{d}, \quad \mathcal{D}_{i} V_{\kappa}=V_{\kappa} \partial_{i}, \quad 1 \leq i \leq d .
$$

The explicit formula of $V_{\kappa}$, however, is known only in the case of the group $\mathbb{Z}_{2}^{d}$ and $S_{3}$, the symmetric group of three variables. For each $\beta \in \mathbb{N}_{0}^{d}$, the function $V_{\kappa} x^{\beta}$ is a homogeneous polynomial of degree $|\beta|$. Define

$$
R_{\beta}(x):=\operatorname{proj}_{n}^{B} V_{\kappa}\left\{x^{\beta}\right\}=V_{\kappa}\left[\sum_{0 \leq j \leq n / 2} \frac{1}{4^{j} j !(-n-\lambda-\mu+1)_{j}} \Delta^{j} x^{\beta}\right],
$$

where the second equality follows from (2.2) and the fact that $\Delta_{h} V_{\kappa}=V_{\kappa} \Delta$. Clearly $R_{\beta}$ is an orthogonal polynomial of degree $n$ with respect to $W_{\kappa, \mu}$ and $\left\{R_{\beta}:|\beta|=n\right\}$ is a basis of $\mathcal{V}_{n}^{d}\left(W_{\kappa, \mu}\right)$. It turns out that $R_{\beta}$ and $U_{\alpha}$ are biorthogonal. For $\alpha \in \mathbb{N}_{0}^{d}$, write $\alpha !=\alpha_{1} ! \ldots \alpha_{d} !$ 
Theorem 2.4. The two families $\left\{U_{\alpha}:|\alpha|=n, \alpha \in \mathbb{N}_{0}^{d}\right\}$ and $\left\{R_{\alpha}:|\alpha|=n, \alpha \in \mathbb{N}_{0}^{d}\right\}$ are biorthogonal with respect to $W_{\kappa, \mu}$ on $B^{d}$; more precisely,

$$
a_{\kappa} \int_{B^{d}} U_{\alpha}(x) R_{\beta}(x) W_{\kappa, \mu}(x) d x=A_{\alpha} \delta_{\alpha, \beta}, \quad A_{\alpha}=(-1)^{n} \alpha ! \frac{(\mu+1 / 2)_{n}}{(\lambda+\mu+1 / 2)_{n}} .
$$

In particular, $\left\{U_{\alpha}:|\alpha|=n\right\}$ is a basis for $\mathcal{V}_{n}^{d}\left(W_{\kappa, \mu}\right)$.

Proof. Since both $U_{\alpha}$ and $V_{\beta}$ are orthogonal polynomials with respect to $W_{\kappa, \mu}$, we only need to consider the case that they are both polynomials of degree $n$. Let $\alpha, \beta \in \mathbb{N}_{0}^{d}$ and $|\alpha|=|\beta|$. Following the proof of Proposition 2.3, we have

$$
\begin{aligned}
& a_{\kappa} \int_{B^{d}} U_{\alpha}(x) R_{\beta}(x) h_{\kappa}^{2}(x)\left(1-\|x\|^{2}\right)^{\mu-1 / 2} d x \\
& =(-1)^{n} a_{\kappa} \int_{B^{d}} \mathcal{D}^{\alpha}\left[R_{\beta}(x)\right]\left(1-\|x\|^{2}\right)^{n+\mu-1 / 2} h_{\kappa}^{2}(x) \\
& =(-1)^{n} a_{\kappa} \int_{B^{d}} \mathcal{D}^{\alpha}\left[V_{\kappa} x^{\beta}\right]\left(1-\|x\|^{2}\right)^{n+\mu-1 / 2} h_{\kappa}^{2}(x) \\
& =\delta_{\alpha, \beta} \alpha !(-1)^{n} a_{\kappa} \int_{B^{d}}\left(1-\|x\|^{2}\right)^{n+\mu-1 / 2} h_{\kappa}^{2}(x),
\end{aligned}
$$

since $R_{\beta}(x)=x^{\beta}+$ lower degree polynomials and $\mathcal{D}^{\alpha} V_{\kappa} x^{\beta}=V_{\kappa} \partial^{\alpha} x^{\beta}=\alpha ! \delta_{\alpha, \beta} V_{\kappa} 1$ $=\alpha ! \delta_{\alpha, \beta}$. The constant is computed by

$$
\begin{aligned}
\int_{B^{d}}\left(1-\|x\|^{2}\right)^{n+\mu-1 / 2} h_{\kappa}^{2}(x) & =\int_{0}^{1} r^{d-1+2 \gamma}\left(1-r^{2}\right)^{\mu-1 / 2} d r \int_{S^{d-1}} h_{\kappa}^{2}\left(x^{\prime}\right) d \omega\left(x^{\prime}\right) \\
& =\frac{\Gamma(\gamma+d / 2) \Gamma(\mu+1 / 2)}{2 \Gamma(\gamma+\mu+(d+1) / 2)} \int_{S^{d-1}} h_{\kappa}^{2}\left(x^{\prime}\right) d \omega\left(x^{\prime}\right) \\
& =\frac{(\mu+1 / 2)_{n}}{(\lambda+\mu+1 / 2)_{n}} a_{\kappa}^{-1},
\end{aligned}
$$

since $a_{\kappa}^{-1}$ is just the above integral with $n=0$. Finally, it follows from the biorthogonality that the set $\left\{U_{\alpha}:|\alpha|=n\right\}$ is linearly independent, so that it is a basis of $\mathcal{V}_{n}^{d}\left(W_{\kappa, \mu}\right)$.

For our next property of $U_{\alpha}(x)$, we will need the reproducing kernel $P_{n}(x, y)$ of $\mathcal{V}_{n}^{d}\left(W_{\kappa, \mu}\right)$. This kernel is characterized by the property that

$$
a_{\kappa} \int_{B^{d}} P(x, y) q(y) W_{\kappa, \mu}(y) d y=q(x), \quad \forall q \in \mathcal{V}_{n}^{d}\left(W_{\kappa, \mu}\right) .
$$

It is shown in 12] that the kernel enjoys a closed formula given by

$$
\begin{aligned}
P_{n}(x, y) & =\frac{n+\lambda+\mu}{\lambda+\mu} c_{\mu} \\
& \times V_{\kappa}\left[\int_{-1}^{1} C_{n}^{\lambda+\mu}\left(\langle x, \cdot\rangle+t \sqrt{1-\|x\|^{2}} \sqrt{1-\|y\|^{2}}\right)\left(1-t^{2}\right)^{\mu-1} d t\right](y),
\end{aligned}
$$

where $c_{\mu}=1 / \int_{-1}^{1}\left(1-t^{2}\right)^{\mu-1} d t$ and $V_{\kappa}$ acts on the variable represented by the dot in the formula. This closed formula for the reproducing kernel has been used to study summability of the Fourier orthogonal expansion on the unit ball. It follows from the biorthogonal property of $\left\{U_{\alpha}\right\}$ and $\left\{R_{\beta}\right\}$ that

$$
P_{n}(x, y)=\sum_{|\alpha|=n} A_{\alpha}^{-1} R_{\alpha}(x) U_{\alpha}(y)
$$


Indeed, multiplying the right-hand side of the above equation by $R_{\beta}(y)$ and integrating, the result is $R_{\beta}(y)$ by the biorthogonality, which shows that the right-hand side satisfies the reproducing property. Since the kernel is unique, it has to equal the left-hand side.

Let $K_{n}(x, y)=V_{\kappa}\left(\langle x, y\rangle^{n}\right) / n$ !. It is known ([3]) that

$$
K_{n}\left(x, \mathcal{D}^{(y)}\right) p(y)=p(x), \quad \forall p \in \mathcal{P}_{n}^{d},
$$

where $\mathcal{D}^{(y)}$ means that $\mathcal{D}_{i}$ is taken with respect to the variable $y$ and $K_{n}\left(x, \mathcal{D}^{(y)}\right)$ is the operator formed by replacing $y_{i}$ by $\mathcal{D}_{i}^{(y)}$ in $K_{n}(x, y)$. A bilinear form $\langle p, q\rangle_{h}$ is defined on $\mathcal{P}_{n}^{d}$ by

$$
\langle p, q\rangle_{h}=p(\mathcal{D}) q(x), \quad p, q \in \mathcal{P}_{n}^{d} .
$$

This form is symmetric in the sense that $\langle p, q\rangle_{h}=\langle q, p\rangle_{h}$ ([3]). We have the following lemma that will be useful below.

Lemma 2.5. Let $\alpha \in \mathbb{N}_{0}^{d}$ with $|\alpha|=n$ and $q \in \mathcal{P}_{j}^{d}$ with $j<n$. Then

$$
\left(\mathcal{D}^{(y)}\right)^{\alpha}\left[q(y) V_{\kappa}^{(y)}\langle x, y\rangle^{n-j}\right]=(n-j) ! q(\mathcal{D}) x^{\alpha} .
$$

Proof. Let $\beta \in \mathbb{N}_{0}^{d}$ and $|\beta|=j$. As a function in $y, y^{\beta} V_{\kappa}\langle x, y\rangle^{n-j}$ is a polynomial in $\mathcal{P}_{n}^{d}$. Using the fact that the bilinear form is symmetric, we get

$$
\begin{aligned}
& \left(\mathcal{D}^{(y)}\right)^{\alpha}\left[y^{\beta} V_{\kappa}\langle x, y\rangle^{n-j}\right] /(n-j) !=\left\langle y^{\alpha}, y^{\beta} K_{n-|\beta|}(x, y)\right\rangle_{h} \\
& =\left\langle y^{\beta} K_{n-|\beta|}(x, y), y^{\alpha}\right\rangle_{h}=K_{n-|\beta|}\left(x, \mathcal{D}^{(y)}\right) \mathcal{D}^{\beta} y^{\alpha}=\mathcal{D}^{\beta} x^{\alpha},
\end{aligned}
$$

where the last step follows from the equation (2.5) with $p(y)=\mathcal{D}^{\beta} y^{\alpha}$ and $n$ replaced by $n-j$.

Proposition 2.6. Let $\alpha \in \mathbb{N}_{0}^{d}$ and $|\alpha|=n$. Then

$$
U_{\alpha}(x)=\sum_{0 \leq 2 j \leq n} \frac{2^{n}(\mu+1 / 2)_{n}}{2^{2 j}(\mu+1 / 2)_{j} j !}(-1)^{n-j}\left(1-\|x\|^{2}\right)^{j} \Delta_{h}^{j} x^{\alpha} .
$$

In particular, $U_{\alpha}(x)=(-1)^{n} 2^{n}(\mu+1 / 2)_{n} x^{\alpha}+\left(1-\|x\|^{2}\right) Q(x)$, where $Q \in \Pi_{n-2}^{d}$.

Proof. Since $\mathcal{D}^{\alpha} R_{\beta}(y)=\alpha ! \delta_{\alpha, \beta}$, applying $\left(\mathcal{D}^{(y)}\right)^{\alpha}$ to the equation (2.4) gives

$$
\alpha ! A_{\alpha}^{-1} U_{\alpha}(x)=\left(\mathcal{D}^{(y)}\right)^{\alpha} P_{n}(x, y) .
$$

We then use the expression of $P_{n}(x, y)$ in (2.3). Since the leading coefficient of $C_{n}^{\lambda}(t)$ is $(\lambda)_{n} 2^{n} / n$ !, it follows that $P_{n}(x, y)$, as a function of $y$, satisfies

$$
\begin{aligned}
P_{n}(x, y)= & \frac{n+\lambda+\mu}{\lambda+\mu} \frac{(\lambda+\mu)_{n} 2^{n}}{n !} c_{\mu} \\
& \times V_{\kappa}\left[\int_{-1}^{1}\left(\langle x, \cdot\rangle+t \sqrt{1-\|x\|^{2}} \sqrt{1-\|y\|^{2}}\right)^{n}\left(1-t^{2}\right)^{\mu-1} d t\right](y)+\ldots \\
= & \frac{(\lambda+\mu+1)_{n} 2^{n}}{n !} c_{\mu} \sum_{0 \leq 2 j \leq n}\left(\begin{array}{c}
n \\
2 j
\end{array}\right) b_{j}\left(1-\|x\|^{2}\right)^{j} V_{\kappa}\left[\left(1-\|y\|^{2}\right)^{j}\langle x, \cdot\rangle^{n-2 j}\right](y)
\end{aligned}
$$

+ polynomial of lower degree in $y$, 
where we have used the binomial formula and the fact that $\int_{-1}^{1} t^{j}\left(1-t^{2}\right)^{\mu-1} d t=0$ if $j$ is odd, and $b_{j}$ is given by

$$
b_{j}=c_{\mu} \int_{-1}^{1} t^{2 j}\left(1-t^{2}\right)^{\mu-1} d t=\frac{(1 / 2)_{j}}{(\mu+1 / 2)_{j}} .
$$

Consequently, since $\mathcal{D}^{\alpha} p(x)=0$ for any $p \in \Pi_{m}^{d}, m<|\alpha|$, we have

$$
\begin{aligned}
\alpha ! A_{\alpha}^{-1} U_{\alpha}(x)=\frac{(\lambda+\mu+1)_{n} 2^{n}}{n !} c_{\mu} & \sum_{0 \leq j \leq n}\left(\begin{array}{c}
n \\
2 j
\end{array}\right) \frac{(1 / 2)_{j}}{(\mu+1 / 2)_{j}}\left(1-\|x\|^{2}\right)^{j} \\
& \times\left(\mathcal{D}^{(y)}\right)^{\alpha}\left[\left(1-\|y\|^{2}\right)^{j} V_{\kappa}\left(\langle x, \cdot\rangle^{n-2 j}\right)(y)\right] .
\end{aligned}
$$

Using Lemma 2.5 with $q(y)=\|y\|^{2 j}$, we have

$$
\begin{aligned}
\left(\mathcal{D}^{(y)}\right)^{\alpha}\left[\left(1-\|y\|^{2}\right)^{j} V_{\kappa}\left(\langle x, \cdot\rangle^{n-2 j}\right)(y)\right] & =(-1)^{j}\left(\mathcal{D}^{(y)}\right)^{\alpha}\left[\|y\|^{2 j} V_{\kappa}\left(\langle x, \cdot\rangle^{n-2 j}\right)(y)\right] \\
& =(-1)^{j}(n-2 j) ! \Delta_{h}^{j} x^{\alpha} .
\end{aligned}
$$

Putting these equations together we conclude

$$
U_{\alpha}(x)=A_{\alpha} \frac{(\lambda+\mu+1)_{n} 2^{n}}{\alpha ! n !} c_{\mu} \sum_{0 \leq j \leq n}(-1)^{j} \frac{n !(1 / 2)_{j}}{(2 j) !(\mu+1 / 2)_{j}}\left(1-\|x\|^{2}\right)^{j} \Delta_{h}^{j} x^{\alpha} .
$$

Using the identity $(2 j) !=2^{2 j}(1 / 2)_{j} j$ ! to simplify the constants completes the proof.

We note that the expansion is still implicit in a way, since the computation of $\Delta_{h}^{j} x^{\alpha}$ can be difficult. In the case of classical orthogonal polynomials on $B^{d}, \Delta_{h}$ becomes $\Delta$, and the multinomial formula gives

$$
\Delta^{j} x^{\alpha}=\left(\partial_{1}^{2}+\ldots+\partial_{d}^{2}\right)^{j} x^{\alpha}=\sum_{|\beta|=j} \frac{j !}{\beta !} \partial^{2 \beta} x^{\alpha}=\sum_{|\beta|=j} \frac{j ! \alpha !}{\beta !(\alpha-2 \beta) !} x^{\alpha-2 \beta},
$$

from which the explicit formula of $U_{\alpha}(x)$ for $W_{\mu}$ follows. Since Dunkl's operators commute, the multinomial formula can also be used to expand $\Delta_{h} x^{\alpha}$. However, we do not have a nice formula for $\mathcal{D}^{\beta} x^{\alpha}$. This is a polynomial of degree $|\alpha|-|\beta|$ in $x$. For $|\alpha|=|\beta|$ it is equal to $\left\langle x^{\alpha}, x^{\beta}\right\rangle_{h}$ and is easily seen to be a polynomial in variable $\kappa$. However, we do not know an explicit formula for this quantity. We mention the following recursive formula, which can be used to compute $\mathcal{D}^{\beta} x^{\alpha}$ of lower degree.

Proposition 2.7. Let $\alpha, \beta \in \mathbb{N}_{0}^{d},|\beta| \leq|\alpha|$. If $\alpha_{i}>0$, then

$$
\mathcal{D}^{\beta} x^{\alpha}=x_{i} \mathcal{D}^{\beta} x^{\alpha-e_{i}}+\beta_{1} \mathcal{D}^{\beta-e_{i}} x^{\alpha-e_{i}}+\sum_{v \in R_{+}} \kappa_{v}\left\langle v, e_{i}\right\rangle P_{\beta, v}(\mathcal{D}) x^{\alpha-e_{i}},
$$

where $P_{\beta, v}(y)=\left(y^{\beta}-\left(y \sigma_{v}\right)^{\beta}\right) /\left\langle y, \sigma_{v}\right\rangle$ is a homogeneous polynomial of degree $|\beta|-1$.

Proof. We use the product rule of Dunkl's operators (4 p. 157])

$$
\mathcal{D}_{i}(f g)(x)=g(x) \mathcal{D}_{i} f(x)+f(x) \partial_{i} g(x)+\sum_{v \in R_{+}} \kappa_{v} f\left(x \sigma_{v}\right)\left\langle v, e_{i}\right\rangle \frac{g(x)-g\left(x \sigma_{v}\right)}{\langle x, v\rangle} .
$$


Using the fact that $\mathcal{D}_{i} K_{n}(x, y)=x_{i} K_{n-1}(x, y)$, the product rule with $g(y)=y^{\beta}$ and $f(y)=K_{|\alpha|-|\beta|}(x, y)$ shows that

$$
\begin{aligned}
\mathcal{D}_{i}^{(y)}\left[y^{\beta} K_{|\alpha|-|\beta|}(x, y)\right]= & y^{\beta} x_{i} K_{|\alpha|-|\beta|-1}(x, y)+K_{|\alpha|-|\beta|}(x, y) \beta_{i} y^{\beta-e_{i}} \\
& +\sum_{v \in R_{+}} \kappa_{v} K_{|\alpha|-|\beta|}\left(x, y \sigma_{v}\right)\left\langle v, e_{i}\right\rangle \frac{y^{\beta}-\left(y \sigma_{v}\right)^{\beta}}{\langle x, v\rangle} .
\end{aligned}
$$

Let $\phi_{\alpha, \beta}(x)=\left(\mathcal{D}_{i}^{(y)}\right)^{\alpha}\left[y^{\beta} K_{|\alpha|-|\beta|}(x, y)\right]$. Since $K_{n}(x w, y w)=K_{n}(x, y)$ for all $w \in G$, applying $\left(\mathcal{D}_{i}^{(y)}\right)^{\alpha-e_{i}}$ to the above equation gives

$$
\phi_{\alpha, \beta}(x)=x_{i} \phi_{\alpha-e_{i}, \beta}(x)+\beta_{i} \phi_{\alpha-e_{i}, \beta-e_{i}}(x)+\sum_{v \in R_{+}} \kappa_{v}\left\langle v, e_{i}\right\rangle \sum_{|\tau|=|\beta|-1} c_{\tau} \phi_{\alpha-e_{i}, \tau}\left(x \sigma_{v}\right),
$$

where $\left(y^{\beta}-\left(y \sigma_{v}\right)^{\beta}\right) /\langle x, v\rangle=\sum_{|\tau|=|\beta|-1} c_{\tau} y^{\tau}$. By Lemma 2.5 $\phi_{\alpha, \beta}(x)=\mathcal{D}^{\beta} x^{\alpha}$, which gives the stated formula.

\section{RELATION TO AN ORTHONORMAL BASIS}

The biorthogonality of two bases is useful for finding the orthogonal expansions of a function. For example, for $f \in L^{2}\left(W_{\kappa, \mu}\right)$, the Fourier orthogonal expansion of $f$ in terms of the basis $\left\{U_{\alpha}\right\}$ is given by

$$
f(x)=\sum_{n=0}^{\infty} \sum_{|\alpha|=n} b_{\alpha, n} A_{\alpha}^{-1} U_{\alpha}(x), \quad b_{\alpha, n}=a_{\kappa} \int_{B^{d}} f(x) R_{\alpha}(x) W_{\kappa, \mu}(x) d x,
$$

where the equality holds in the $L^{2}$ norm. That the coefficient $b_{\alpha, n}$ is given as above can be easily seen upon integrating the expansion of $f(x) R_{\alpha}(x)$ over $B^{d}$. Thus, the biorthogonality provides an easy way to compute the Fourier coefficient $b_{\alpha, n}$ in the expansion with respect to $U_{\alpha}(x)$. Note that $R_{\alpha}$ depends on the intertwining operator $V_{\kappa}$. The following formula proved in [1] is useful for computing integrals of $V_{\kappa} f$, which works despite the lack of an explicit formula for $V_{\kappa}$.

Lemma 3.1. For a continuous function $f$ on $B^{d}$,

$$
\int_{S^{d-1}} V_{\kappa} f(x) h_{\kappa}^{2}(x) d \omega(x)=B_{\kappa} \int_{B^{d}} f(x)\left(1-\|x\|^{2}\right)^{\gamma-1} d x
$$

where the constant $B_{\kappa}$ can be determined by setting $f(x)=1$.

We use this lemma to work out the expansion in one particular case. Let $C_{n}^{(\mu, \tau)}(t)$ be polynomials defined by the generating function

$$
c_{\mu} \int_{-1}^{1} \frac{1}{\left(1-2 x t+t^{2}\right)^{\mu}}(1+t)\left(1-t^{2}\right)^{\tau-1} d t=\sum_{n=0}^{\infty} C_{n}^{(\mu, \tau)}(x) t^{n} .
$$

These are orthogonal polynomials with respect to the weight function $w_{\mu, \tau}(t)=$ $|t|^{2 \tau}\left(1-t^{2}\right)^{\mu-1 / 2}$ on $[-1,1]$ and they are related to the Jacobi polynomials $P_{n}^{(\alpha, \beta)}(t)$. In particular,

$$
C_{2 n}^{(\mu, \tau)}(t)=\frac{(\mu+\tau)_{n}}{(\tau+1 / 2)_{n}} P_{n}^{(\mu-1 / 2, \tau-1 / 2)}\left(2 t^{2}-1\right) .
$$

It is known that an orthonormal basis of $\mathcal{V}_{n}^{d}\left(W_{\kappa, \mu}\right)$ is given by

$$
\left\{f_{j, \beta}(x):=m_{j} \widetilde{C}_{2 j}^{(\mu, n-2 j+\lambda)}(\|x\|) S_{\beta}^{h}(x): S_{\beta}^{h} \in \mathcal{H}_{n-2 j}^{d}\left(h_{\kappa}^{2}\right), \quad 0 \leq 2 j \leq n\right\},
$$


in which $c_{j}^{-2}$ is the $L^{2}\left(w_{\lambda, \mu},[-1,1]\right)$ norm of $C_{2 j}^{(\mu, n-2 j+\lambda)}(t)$ and $\left\{S_{\beta}^{h}\right\}$ is an orthonormal basis of $\mathcal{H}_{n-2 j}^{d}\left(h_{\kappa}^{2}\right)$. In particular, the polynomial $C_{2 n}^{(\mu, \lambda)}(\|x\|)$ is an element of $\mathcal{V}_{2 n}^{d}\left(W_{\kappa, \mu}\right)$; hence it can be written in terms of $U_{\alpha}(x)$.

Proposition 3.2. The following expansion holds:

$$
\frac{C_{2 n}^{(\mu, \lambda)}(\|x\|)}{C_{2 n}^{(\mu, \lambda)}(1)}=\frac{n !}{(\mu+1 / 2)_{2 n} 2^{2 n}} \sum_{|\beta|=n} \frac{1}{\beta !} U_{2 \beta}(x) .
$$

Proof. The fact that $\left\{U_{\alpha}:|\alpha|=2 n\right\}$ is a basis of $\mathcal{V}_{2 n}^{d}\left(W_{\kappa, \mu}\right)$ shows that we can write

$$
C_{2 n}^{(\mu, \lambda)}(\|x\|)=\sum_{|\alpha|=2 n} b_{\alpha} A_{\alpha}^{-1} U_{\alpha}(x) .
$$

Using the biorthogonality in Proposition 2.4 and the fact that $R_{\kappa}(x)=V_{\kappa} x^{\alpha}+\ldots$, we can compute the coefficient $b_{\alpha}$ as follows:

$$
\begin{gathered}
b_{\alpha}=a_{\kappa, \mu} \int_{B^{d}} C_{2 n}^{(\mu, \lambda)}(\|x\|) R_{\alpha}(x) h_{\kappa}^{2}(x)\left(1-\|x\|^{2}\right)^{\mu-1} d x \\
=a_{\kappa, \mu} \int_{B^{d}} C_{2 n}^{(\mu, \lambda)}(\|x\|) V_{\kappa}\left[x^{\alpha}\right] h_{\kappa}^{2}(x)\left(1-\|x\|^{2}\right)^{\mu-1} d x \\
=b_{\kappa, \mu} \int_{0}^{1} r^{2 n+d-1+2 \gamma} C_{2 n}^{(\mu, \lambda)}(r)\left(1-r^{2}\right)^{\mu-1 / 2} d r \\
\cdot c_{h} \int_{S^{d-1}} V_{\kappa}\left(\{\cdot\}^{\alpha}\right)\left(x^{\prime}\right) h_{\kappa}^{2}\left(x^{\prime}\right) d \omega\left(x^{\prime}\right),
\end{gathered}
$$

using the polar coordinates $x=r x^{\prime}, r>0$ and $x^{\prime} \in S^{d-1}$, where

$$
b_{\kappa, \mu}=1 / \int_{0}^{1} r^{2 \lambda}\left(1-r^{2}\right)^{\mu-1 / 2} d r
$$

and

$$
c_{h}=1 / \int_{S^{d-1}} h_{\kappa}^{2}\left(x^{\prime}\right) d \omega\left(x^{\prime}\right)
$$

The first integral can be computed using the $L^{2}$ norm and the leading coefficient $k_{n}^{(\mu, \tau)}$ of $C_{n}^{(\mu, \tau)}(t)$ (cf. [4, p. 27]),

$$
\begin{aligned}
& b_{\kappa, \mu} \int_{0}^{1} r^{2 n+d-1+2 \gamma} C_{2 n}^{(\mu, \lambda)}(r)\left(1-r^{2}\right)^{\mu-1 / 2} d r \\
& =b_{\kappa, \mu}\left(k^{(\mu, \tau)}\right)^{-1} \int_{0}^{1}\left[C_{2 n}^{(\mu, \lambda)}(r)\right]^{2}\left(1-r^{2}\right)^{\mu-1 / 2} d r=\frac{(\mu+1 / 2)_{n}(\lambda+\mu)_{n}}{(\lambda+\mu+1)_{2 n}}
\end{aligned}
$$

The integral of $V_{\kappa} x^{\alpha}$ can be computed using Lemma 3.1

$$
c_{h} \int_{S^{d-1}} V_{\kappa}\left(\{\cdot\}^{\alpha}\right)\left(x^{\prime}\right) h_{\kappa}^{2}\left(x^{\prime}\right) d \omega=a_{\kappa} \int_{B^{d}} x^{\alpha}\left(1-\|x\|^{2}\right)^{\gamma-1} d x
$$

where $a_{\kappa}=1 / \int_{B^{d}}\left(1-\|x\|^{2}\right)^{\gamma-1} d x$, which shows that the integral is zero if one of $\alpha_{i}$ is odd. If $\alpha=2 \beta$, then the integral over $B^{d}$ can be evaluated using polar coordinates and the result is

$$
c_{h} \int_{S^{d-1}} V_{\kappa}\left(\{\cdot\}^{\alpha}\right)\left(x^{\prime}\right) h_{\kappa}^{2}\left(x^{\prime}\right) d \omega=\frac{(1 / 2)_{\beta}}{(\lambda+1 / 2)_{\beta}} .
$$


Putting these equations together, we have proved that

$$
C_{2 n}^{(\mu, \lambda)}(\|x\|)=\sum_{|\beta|=n} \frac{(\mu+1 / 2)_{n}(\mu+\lambda)_{n}}{(\mu+\lambda+1)_{2 n}} \frac{(1 / 2)_{\beta}}{(\lambda+1 / 2)_{\beta}} A_{2 \beta}^{-1} U_{2 \beta}(x) .
$$

Using the formula of $C_{2 n}^{(\mu, \lambda)}(1)$ (4, p. 27]) and simplifying the coefficients completes the proof.

Let us write the expansion explicitly. Using the multinomial formula and the Rodrigues type formula of $U_{\beta}(x)$, we see that the equation (3.3) gives

$$
\begin{aligned}
\frac{C_{2 n}^{(\mu, \lambda)}(\|x\|)}{C_{2 n}^{(\mu, \lambda)}(1)} & =\frac{1}{(\mu+1 / 2)_{2 n} 2^{2 n}}\left(1-\|x\|^{2}\right)^{-\mu+1 / 2} \sum_{|\beta|=n} \frac{n !}{\beta !} \mathcal{D}^{2 \beta}\left(1-\|x\|^{2}\right)^{2 n+\mu-1 / 2} \\
& =\frac{1}{(\mu+1 / 2)_{2 n} 2^{2 n}}\left(1-\|x\|^{2}\right)^{-\mu+1 / 2} \Delta_{h}^{n}\left(1-\|x\|^{2}\right)^{2 n+\mu-1 / 2}
\end{aligned}
$$

In particular, if $\kappa=0$ and $d=1$, so that $\lambda=0$, then $C_{2 n}^{(\mu, 0)}(\|x\|)$ becomes the Gegenbauer polynomial $C_{2 n}^{\mu}(x)$ and the above formula is precisely the Rodrigues formula for the Gegenbauer polynomials. Furthermore, in polar coordinates, the $h$-Laplacian can be written as

$$
\Delta_{h}=\frac{\partial^{2}}{\partial r^{2}}+\frac{2 \lambda+1}{r} \frac{\partial}{\partial r}+\frac{1}{r^{2}} \Delta_{h, 0}
$$

where $\Delta_{h, 0}$ is the spherical part of the operator which has $h$-harmonics as eigenfunctions. More precisely, if $S_{n}^{h} \in \mathcal{H}_{n}^{d}\left(h_{\kappa}^{2}\right)$, then

$$
\Delta_{h, 0} S_{n}^{h}(x)=-n(n+2 \lambda) S_{n}^{h}(x) .
$$

This leads to the following Rodrigues type formula for $C_{2 n}^{(\mu, \lambda)}(t)$.

Proposition 3.3. For $n \geq 0$,

$$
\frac{C_{2 n}^{(\mu, \lambda)}(r)}{C_{2 n}^{(\mu, \lambda)}(1)}=\frac{1}{(\mu+1 / 2)_{2 n} 2^{2 n}}\left(1-r^{2}\right)^{-\mu+1 / 2}\left(\frac{d^{2}}{d r^{2}}+\frac{2 \lambda}{r} \frac{d}{d r}\right)^{n}\left(1-r^{2}\right)^{2 n+\mu-1 / 2} .
$$

By the relation (3.1), this gives a Rodrigues type formula for the Jacobi polynomials, which can be rewritten as

$$
P_{n}^{(\alpha, \beta)}\left(2 r^{2}-1\right)=\frac{(\alpha+1)_{n}}{(\alpha+1)_{2 n} n ! 2^{2 n}}\left(1-r^{2}\right)^{-\alpha}\left(\frac{d^{2}}{d r^{2}}+\frac{2 \beta+1}{r} \frac{d}{d r}\right)^{n}\left(1-r^{2}\right)^{2 n+\alpha} .
$$

In fact, this formula was discovered by Koornwinder [6] in the course of giving an analytic proof for his addition formula for the Jacobi polynomials.

It is possible to derive a Rodrigues type formula for other elements in the orthonormal basis (3.2). We have the following.

Proposition 3.4. Let $S_{n-2 j}^{h}$ be an h-spherical harmonic in $\mathcal{H}_{n}^{d}\left(h_{\kappa}^{2}\right)$. Then

$$
\begin{aligned}
& \frac{C_{2 j}^{(\mu, n-2 j+\lambda)}(\|x\|)}{C_{2 j}^{(\mu, n-2 j+\lambda)}(1)} S_{n-2 j}^{h}(x) \\
& =\frac{1}{(\mu+1 / 2)_{2 j} 2^{2 j}}\left(1-\|x\|^{2}\right)^{-\mu+1 / 2} \Delta_{h}^{j}\left[\left(1-\|x\|^{2}\right)^{2 j+\mu-1 / 2} S_{n-2 j}^{h}(x)\right] .
\end{aligned}
$$


Proof. Let $g$ be a differentiable function defined on $[0,1]$ and $S_{m}^{h} \in \mathcal{H}_{m}^{d}\left(h_{\kappa}^{2}\right)$. We claim that

$$
\Delta_{h}\left[g(\|x\|) S_{m}^{h}(x)\right]=S_{m}^{h}(x)\left(\frac{d^{2}}{d r^{2}}+\frac{2 \lambda+2 m+1}{r} \frac{d}{d r}\right) g(r), \quad r=\|x\| .
$$

Indeed, since $S_{m}^{h}$ is a homogeneous polynomial of degree $r, S_{m}^{h}(x)=r^{m} S_{m}^{h}\left(x^{\prime}\right)$ under the polar coordinates. Therefore, using (3.4) and (3.5),

$$
\begin{aligned}
\Delta_{h}\left[g(\|x\|) S_{m}^{h}(x)\right]= & \left(\frac{d^{2}}{d r^{2}}+\frac{2 \lambda+1}{r} \frac{d}{d r}\right)\left(r^{m} g(r)\right) \cdot S_{m}^{h}\left(x^{\prime}\right) \\
& -m(m+2 \lambda) g(r) r^{m-2} S_{m}^{h}\left(x^{\prime}\right) \\
= & {\left[\left(\frac{d^{2}}{d r^{2}}+\frac{2 \lambda+2 m+1}{r} \frac{d}{d r}\right) g(r)\right] r^{m} S_{m}^{h}\left(x^{\prime}\right), }
\end{aligned}
$$

where the second equality follows from a simple computation. Clearly, we can use the formula repeatedly to get

$$
\Delta_{h}^{j}\left[g(\|x\|) S_{m}^{h}(x)\right]=S_{m}^{h}(x)\left(\frac{d^{2}}{d r^{2}}+\frac{2 \lambda+2 m+1}{r} \frac{d}{d r}\right)^{j} g(r) .
$$

Applying the above formula with $g(r)=\left(1-r^{2}\right)^{2 j+\mu-1 / 2}$ and $m=n-2 j$, the stated formula follows from Proposition 3.3 .

\section{ACKNOWLEGEMENT}

The author thanks a referee for his/her careful review.

\section{REFERENCES}

[1] P. Appell and J. K. de Fériet, Fonctions hypergéométriques et hypersphériques, Polynômes d'Hermite, Gauthier-Villars, Paris, 1926.

[2] C. Dunkl, Differential-difference operators associated to reflection groups, Trans. Amer. Math. Soc. 311 (1989), 167-183. MR0951883 (90k:33027)

[3] C. Dunkl, Integral kernels with reflection group invariance, Canad. J. Math. 43 (1991), 12131227. MR 1145585 (93g:33012)

[4] C. F. Dunkl and Yuan Xu, Orthogonal polynomials of several variables, Cambridge Univ. Press, 2001. MR1827871 (2002m:33001)

[5] A. Erdélyi, W. Magnus, F. Oberhettinger and F. G. Tricomi, Higher transcendental functions, McGraw-Hill, Vol 2, New York, 1953. MF0058756 (15:419i)

[6] T. Koornwinder, Jacobi polynomials, III. An analytic proof of the addition formula, SIAM J. Math. Anal., 6 (1975), pp. 533-543. MR0447659 (56:5969)

[7] L. Lapointe and L. Vinet, Rodrigues formulas for the Macdonald polynomials, Adv. Math. 130 (1997), 261-279. MR 1472319|(99e:05128)

[8] A. Nishino, H. Ujino and M. Wadati, Rodrigues formula for the nonsymmetric multivariable Laguerre polynomial, J. Phys. Soc. Japan 68 (1999),797-802. MF,1697908 (2000c:33014)

[9] G. Szegő, Orthogonal polynomials, 4th ed., American Mathematical Society Colloquium Publication 23, American Mathematical Society, Providence, RI, 1975. MR0372517 (51:8724)

[10] H. Ujino and M. Wadati, Rodrigues formula for the nonsymmetric multivariable Hermite polynomial, J. Phys. Soc. Japan 68 (1999), 391-395. MR1682683 (2000g:33016)

[11] Yuan Xu, Integration of the intertwining operator for $h$-harmonic polynomials associated to reflection groups, Proc. Amer. Math. Soc. 125 (1997), 2963-2973. MR1402890 (97m:33004) 
[12] Yuan $\mathrm{Xu}$, Orthogonal polynomials on the ball and on the simplex for weight functions with reflection symmetries, Constr. Approx., 17 (2001), 383-412. MR1828918 (2002e:33018)

[13] Yuan Xu, Orthogonal polynomials and summability in Fourier orthogonal series on spheres and on balls, Math. Proc. Cambridge Phil. Soc. 31 (2001), 139-155. MR1833079 (2002d:42031)

Department of Mathematics, University of Oregon, Eugene, Oregon 97403-1222

E-mail address: yuan@math.uoregon.edu 